\title{
SYSTEM FOR MEASURING KINEMATICS OF VESTIBULAR SYSTEM MOVEMENTS IN NEUROLOGICAL PRACTICE
}

\author{
Petr $\operatorname{VolF}^{a, *}$, Patrik Kutílek ${ }^{a}$, Jiří Hozman ${ }^{a}$, Rudolf Černý ${ }^{b}$, \\ TOMÁš KOUKOLÍK ${ }^{a}$, JAN HeJdA ${ }^{a}$ \\ ${ }^{a}$ Faculty of Biomedical Engineering, Czech Technical University in Prague, nám. Sítná 3105, 27201 Kladno, \\ Czech Republic \\ ${ }^{b}$ 2nd Faculty of Medicine - Motol University Hospital, Charles University in Prague, V Úvalu 84,15006 \\ Praha 5, Czech Republic \\ * corresponding author: petr.volf@fbmi.cvut.cz
}

\begin{abstract}
The article deals with the design of a system for studying kinematics of movement of the vestibular system. Up to now there has not existed a system which would enable to measure the kinematic quantities of movement of the individual parts of the vestibular system within its coordinate system. The proposed system removes these deficiencies by suitable positioning of five gyro-accelerometric units on the helmet. The testing of the system took place under two conditions, during Unilateral Rotation on Barany Chair and Head Impulse Test. During the testing, the system justified its application because the results show that the kinematic quantities of the movement of the left and right labyrinths of the vestibular system differ. The introduced device is mainly intended for application in clinical neurology with the aim to enable the physician to measure all linear and angular accelerations of the vestibular system during medical examinations.
\end{abstract}

KEYWORDS: kinematics, vestibular system, head movement, gyroscope, accelerometer.

\section{INTRODUCTION}

Abnormal head posture is an important clinical sign of disease in many medical areas 1 . There is a large number of neurological disorders that affect the postural alignment of the head. The simultaneous measurement of the vestibular system movements could contribute to better understanding of diseases affecting the vestibular system functions[2]. Currently there is a number of proposed methods of measurement and evaluation of the position of the head where the abnormalities in position can be caused by the dysfunction of the vestibular system [3] [4]. Nevertheless, the majority of systems enable us to measure only the movement of the head or of other segments of the body but does not allow us to measure the movement of the vestibular system as such or the movement of the inner ear. Even though these systems are commonly used for vestibular system examinations, they are not able to detect its movements directly. These are for example the EyeSeeCam glasses equipped with gyro-accelerometers for measuring movement of the head [5], or possibly a helmet with gyro-accelerometers for medical research of the vestibular system [6]. The accelerometric system for indirect examination of the vestibular system was also used in the system for electrotactile vestibular stimulation [7]. In this case, the accelerometer was placed on the superior side of the tongue. For similar examination, other systems with gyro-accelerometers built in helmets or in mouth pieces can potentially be used for measuring the movement of the head $[8]$. For purposes of pros- thetic substitution of the vestibular function, gyroaccelerometric systems are being developed which are based on the cochlear implant stimulating the afferent activity of ampular fibers of the individual semicircular canals [9] 10]. Accelerometers placed in the helmet or rather on the surface of the helmet are used to measure the impact to the head within the study of the head load during sport activities such as American football, hockey or baseball[11]8. In some cases of head movement measurement a great number of accelerometers is used (10 and more)[12, but without the possibility to measure the kinematic quantities of movement of the vestibular system within its coordinate system.

Alternatively, to the accelerometric system, the special camera systems developed for measuring the movement of the head in space could be used to measure the movement of the vestibular system 13 . However, none of the mentioned systems or applications use sensors or head placed markers to evaluate the actual complex movement of the vestibular system. Due to their position and measurement, the abovementioned systems do not allow recording the actual movement of the vestibular system because the position of movement sensors is outside the vestibular system of a man. The reason is that these systems are developed to record the movement of anatomical landmarks which are on the surface of the head. Another reason for the impossibility to measure the complex movements of the vestibular system is that the systems have only one sensor or that the construction of the system does not allow for the measurement 
of the movement of the vestibular system. Another disadvantage of the camera systems is the need for the distribution of tracking elements of the system, i.e. cameras of the camera system. These systems do not allow measuring more extensive movement and are expensive in comparison with the gyroscopic or accelerometric systems. The second disadvantage of these systems is the impossibility to track complex 3 dimensional movements due to the possibility of covering up of the marker [14.

However, physicians require measuring all linear and angular movements of the vestibular system only, but not of other segments or anatomical landmarks of the body. It is the knowledge of the movement of part of the vestibular system which is induced by the external stimulus or rather by the methodology of measurement of the function of the vestibular system which is interpreted by the analysis of the kinematic data of the record of the movement. In clinical neurology the stimuli are for example unilateral rotation on the rotating chair [15, HIT (Head Impulse Test) [16] [5], VAT (Vestibular Autorotation test) [17] and others. The mentioned deficiencies should be solved by the newly proposed system which enables us to measure the movement of the vestibular system but not of the head as such during the above mentioned tests. Because the existing systems do not allow for such a complex recording of the movement, the aim of the article is to introduce the new system enabling us to measure the translational and rotational movements of the vestibular system of a man in all 3 axes of the $3-\mathrm{D}$ space. For this purpose, the method of presentation of the evaluation of the bilateral data, i.e. data from the left and right labyrinths of the vestibular system will be shown.

\section{Methods}

\subsection{MEASURING SYSTEM}

The disadvantages of measuring by the existing methods are removed to a great extent by a device for measuring the movement of the vestibular system proposed by us. Its principle is that it consists of a helmet which can be placed on the head of the patient and which is by its construction designed to attach 5 gyro-accelerometric units in such a way that they enable us to precisely measure the movements of the vestibular system see Fig. 1. During the designing process it was supposed that the translational and rotational movements of the vestibular system differ, which means that it was necessary to place the individual gyro-accelerometric units on the anatomical axes of the vestibular system or rather on the axes on which the specific translational and rotational movements are being accomplished.

The designed helmet is equipped with five sliding plates enabling precise positioning of five gyroaccelerometric units, see Fig. 2 and Fig. 3 The sliding plates are placed on the helmet in such a

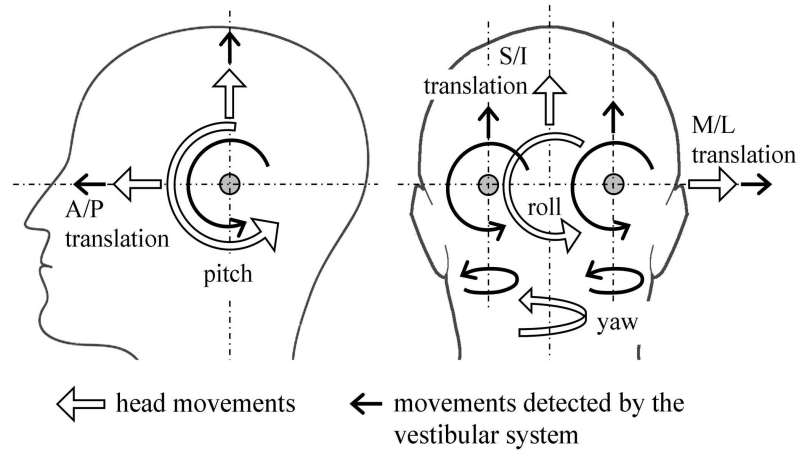

Figure 1. Position of the left and right labyrinths of the vestibular system and the head movements and movements detected by the vestibular system; $\mathrm{A} / \mathrm{P}$ - anterior/posterior, M/L - medial/lateral, S/I superior/inferior [18].

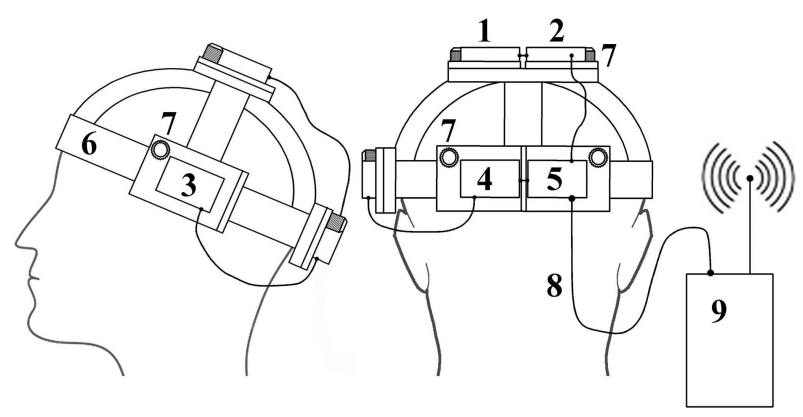

Figure 2. Scheme of the designed system for measuring the movement of the vestibular system: $1-$ sliding plate parietal left with fixed parietal left gyroaccelerometer; 2 - sliding plate parietal right with fixed parietal right gyro-accelerometer; 3 - sliding plate temporal with fixed temporal gyro-accelerometer; 4 - sliding plate occipital left with fixed occipital left gyro-accelerometer; 5 - sliding plate occipital right with fixed occipital right gyro-accelerometer; 6 - support structure of the helmet; 7 - locking screws; 8 - communication cable; 9 - wireless communication unit.

way that their planes of movement correspond with the individual anatomical planes (frontal, transversal, sagittal) of the head in upright position. The gyro-accelerometric units are appropriately placed on each sliding plate so as to enable precise positioning of the gyro-accelerometric units on the anatomical axes passing through the vestibular system. The precise position of the sliding plates on the helmet is secured by locking screws before the measurement.

The sliding plate occipital left is placed in the frontal plane with the center on the axis of the left labyrinth of the vestibular system which is perpendicular to the frontal plane. The sliding plate occipital right is placed in the frontal plane with the center on the axis of the right labyrinth of the vestibular system that is perpendicular to the frontal plane. The sliding plate parietal left is placed in the transversal plane with the center on the axis of the left labyrinth of the vestibular 


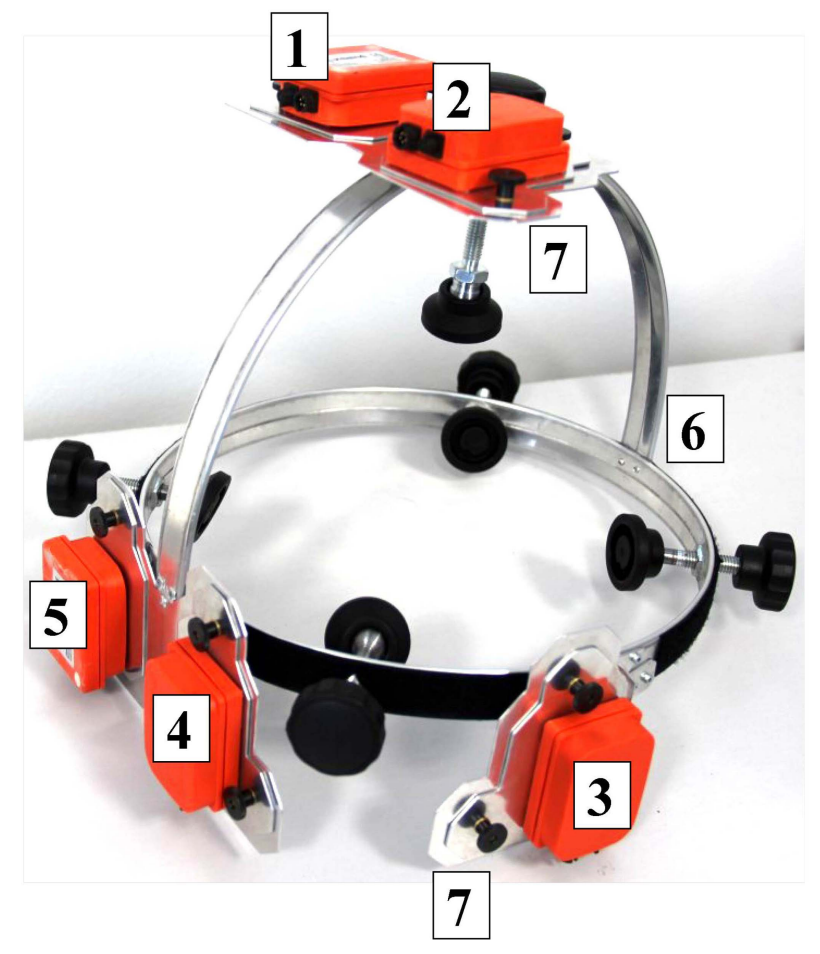

FiguRE 3. Scheme of the designed system for measuring the movement of the vestibular system: $1-$ sliding plate parietal left with fixed parietal left gyroaccelerometer; 2 - sliding plate parietal right with fixed parietal right gyro-accelerometer; 3 - sliding plate temporal with fixed temporal gyro-accelerometer; 4 - sliding plate occipital left with fixed occipital left gyro-accelerometer; 5 - sliding plate occipital right with fixed occipital right gyro-accelerometer; 6 - support structure of the helmet; 7 - locking screws; 8 communication cable.

system that is perpendicular to the transversal plane. The sliding plate parietal right is in the transversal plane with the center on the axis of the right labyrinth of the vestibular system which is perpendicular to the transversal plane. The sliding plate temporal lies in the sagittal plane with its center on the axis of the left and right labyrinths of the vestibular system which is perpendicular to the sagittal plane. The sliding plate occipital left, sliding plate occipital right, sliding plate parietal left, sliding plane parietal right, sliding plate temporal allow continuous movement in any direction of planes of the plates in the extent of $1.5 \mathrm{~cm}$ and their subsequent fixation by the locking screws to the helmet.

The units of the gyro-accelerometer are IMU MTx Xsens containing a 3 axis accelerometer, a 3 axis gyroscope and a 3 axis magnetometer. The gyroaccelerometric unit occipital left is attached to the sliding plate occipital left. The gyro-accelerometric unit occipital right is attached to the sliding plate occipital right. The gyro-accelerometric unit parietal left is attached to the sliding plate parietal left. The gyroaccelerometric unit parietal right is attached to the

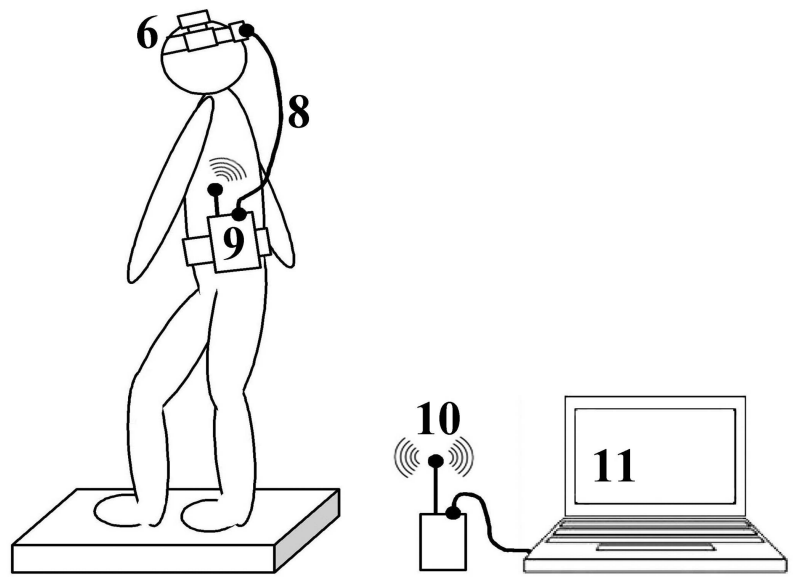

Figure 4. Scheme of the use of the designed system for measuring the movement of the vestibular system: 6 - support structure of the helmet; 8 - communication cable; 9 - wireless communication unit; 10 - receiver of the wireless communication unit 11 - portable PC.

sliding plate parietal right. The gyro-accelerometric unit temporal is attached to the temporal sliding plate. Data from the gyro-accelerometric units are transmitted to the transmitter of the wireless communication unit. The transmitter of the wireless communication unit transmits data to the receiver of the wireless communication unit. The receiver of the wireless communication unit sends data to the $\mathrm{PC}$ which processes the data concerning the movement of the vestibular system see Fig. 4. The units are connected in series with a WR-A Xsens transmitter by the wireless communication unit. The receiver of the wireless communication - XbusMaster Xsens which transmits the measured data to the $\mathrm{PC}$ which processes it by means of a standard data cable USB-A type.

The system is designed to enable repeated measurements of patients with various sizes and profiles of the head. The construction of the helmet is rigid and ensures that the sliding plate occipital left and the sliding plate occipital right are perpendicular to the sliding plate parietal left, the sliding plate parietal right and the sliding plate temporal. The sliding plate parietal left and sliding plate parietal right are simultaneously perpendicular to the temporal sliding plate, see Fig. 2. The system can be operated by one examining person who arranges the fixation of the helmet on the head and the positioning of the sliding plate so that the sensors of the units are on the appropriate anatomical landmarks of the head of the patient which are defined as a perpendicular projection of the ax is of the vestibular system to the appropriate anatomical planes. The sliding plate occipital left must be arranged so that the gyro-accelerometric unit is on the axis of the left labyrinth of the vestibular system which is perpendicular to the frontal plane. The sliding plate occipital right must be arranged so that the gyro-accelerometric unit occipital right is in the axis of the right labyrinth of the vestibular 
system which is perpendicular to the frontal plane. The sliding plate parietal left must be arranged so that the gyro-accelerometric unit parietal left is in the axis of the left labyrinth of the vestibular system which is perpendicular to the transversal plane. The sliding plate parietal right must be arranged so that the gyro-accelerometric unit parietal right is in the axis of the right labyrinth of the vestibular systems which is perpendicular to the transversal plane. The sliding plate temporal must be arranged so that the gyro-accelerometric unit temporalis is in the axis of the left and right labyrinths of the vestibular systems perpendicular to the sagittal plane.

\subsection{MeAsurement AND TESTING}

To test the utilization appropriatness of the proposed system designed for independent measurement of the right and left labyrinth of the vestibular system which hinders the construction compared to other existing systems, we have performed a comparison of data corresponding with the left and right labyrinth of the vestibular system. We assume that the values of accelerations will differ. We use pairs of gyroaccelerometers in occipital and parietal parts of a head. For further testing of the system we selected an occipital pair that allows us to measure linear accelerations in the anterior-posterior direction and simultaneously angular acceleration corresponding with roll angular movement in the frontal plane. These movements are commonly studied for example to assess body responses to translational movement [6]. Gyroaccelerometric sensors were set up so that their axes lie on the axes of the vestibular system which pass through its center. These axes are then perpendicular to the frontal plane and the rotation movement around these axes matches the roll movement of the vestibular system see Fig. 1. A healthy subject (age 22) was selected for this testing and two types of movements were performed. The HIT (Head Impulse test) [16] [5] was carried out repeatedly with the rotation around the vertical axis of the body and unilateral rotation on the rotation chair 15 within a 1 minute period when the longitudinal axis of the body lay on the axis of chair rotation. Within the tests we measured kinematic data of the movement of the vestibular system, namely linear acceleration in the frontal direction of a head and angular acceleration corresponding with the head roll movement. The values of these kinematic quantities are output data from the used MTx Xsens gyro-accelerometers. The sensor fusion algorithm (Extended Kalman Filter) was implemented in the unit [19]. The sensors signal generation model is described directly by Xsens, see also 20. The output signal was not filtered in any other way, to have the possibility of considering errors in the final evaluation. The sway was measured with precision of $<0.5^{\circ}$, the linear acceleration was measured with precision of $<0.02 \mathrm{~m} \mathrm{~s}^{-2}$, and the sampling frequency was $100 \mathrm{~Hz}$.
Since the vestibular system records accelerations, it was necessary to convert the angular velocities to angular accelerations. Derivation of the recorded angular velocity was carried out in the MatLab environment (MathWorks, Inc.). For the realization of numeric derivation we used the DIFF function which calculates the differences between adjacent elements of the angular velocity record $[21]$.

After determination of two linear accelerations of the right and left labyrinth of the vestibular system and two angular accelerations of the right and left labyrinth of the vestibular system, the presentation of data, using time dependencies that are commonly used for studies of minimal, or maximal values of kinematic quantities 6, was performed. Furthermore, we employed so-called bilateral diagrams of mutual dependencies of development of identical kinematic quantities for the right and left body part 22 . For our purposes we will presume a presentation of kinematic quantities of movements of parts of the vestibular system (left and right labyrinth) in a 2-D chart. In the case of perfect symmetry, all the chart points of both simultaneously recorded quantities (for the right and left body part) would lie on the axis passing through the beginning of the chart and would be at an angle of $45^{\circ}$. To be able to compare the symmetry in the case of the tests during which the head rotation occurs (on the rotation chair with the axis identical to the longitudinal axis of the body and HIT), it was necessary, with one of the accelerations of translational movement (in our case the right labyrinth of the vestibular system), to convert the development of the values of the signal (multiply by -1 ). This way of presentation of kinematic quantities has not been used so far in connection with the vestibular system.

\subsection{StATISTIC ASSESSMENT}

The comparison of kinematic data of the left and right labyrinth of the vestibular system has furthermore been carried out. The Spearman's rank correlation coefficient between the kinematic data of the left and right vestibular system was calculated to study the differences between the data of left and right IMU. Spearman's rank correlation coefficient was employed since this regards a nonparametric method which uses the ranking of values of the quantitites observed and thus it does not require normality of data. The advantage lies in the possibility to use this method for description of any linear or nonlinear dependencies 23 . Spearman's correlation coefficient is thus used for measurements of the power of the relationship with such quantities where it is not possible to assume the linearity of the expected correlation or normal division of the observed variables. If the value of the Spearman's rank correlation coefficient falls between $0.0-0.4$, there is a weak correlation, $0.4-0.6$, there is a moderate correlation, $0.6-1.0$, there is a strong correlation. 

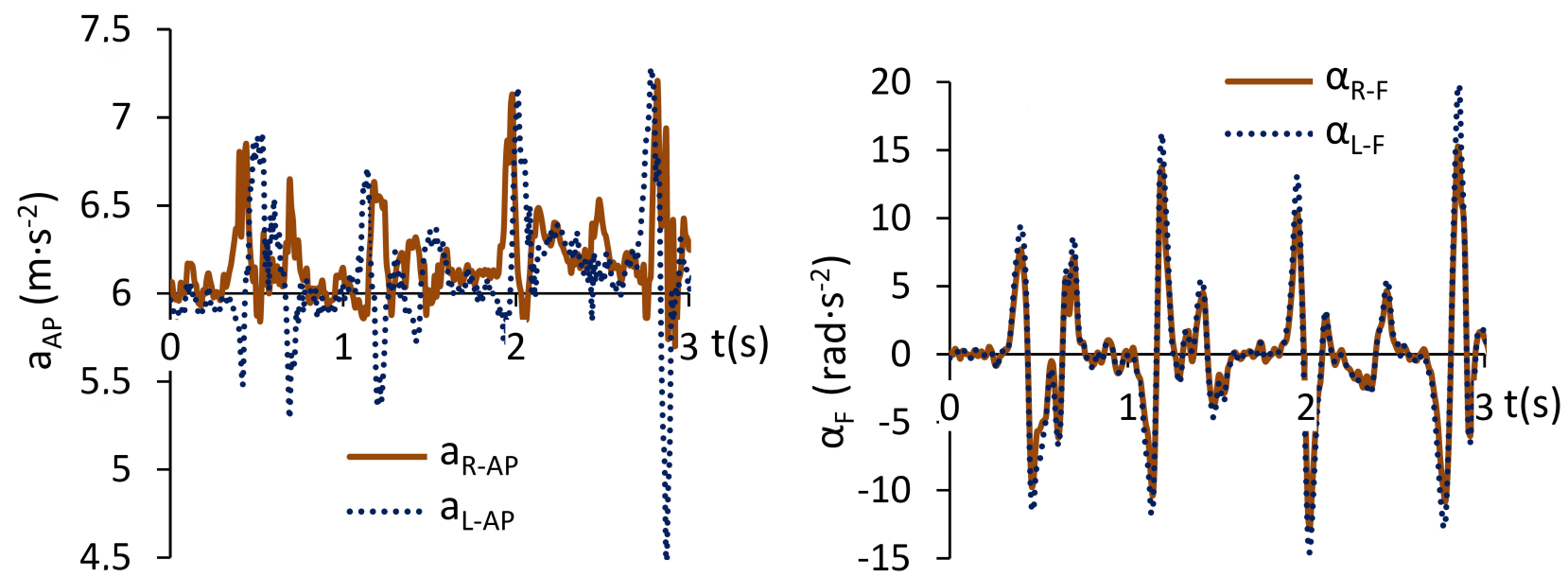

FigURE 5. Linear accelerations chart (a) in the anterior-posterior (AP) direction and angular accelerations $(\alpha)$ in the frontal plane $(\mathrm{F})$ of the left $(\mathrm{L})$ and right $(\mathrm{R})$ labyrinth of the vestibular system measured within HIT.
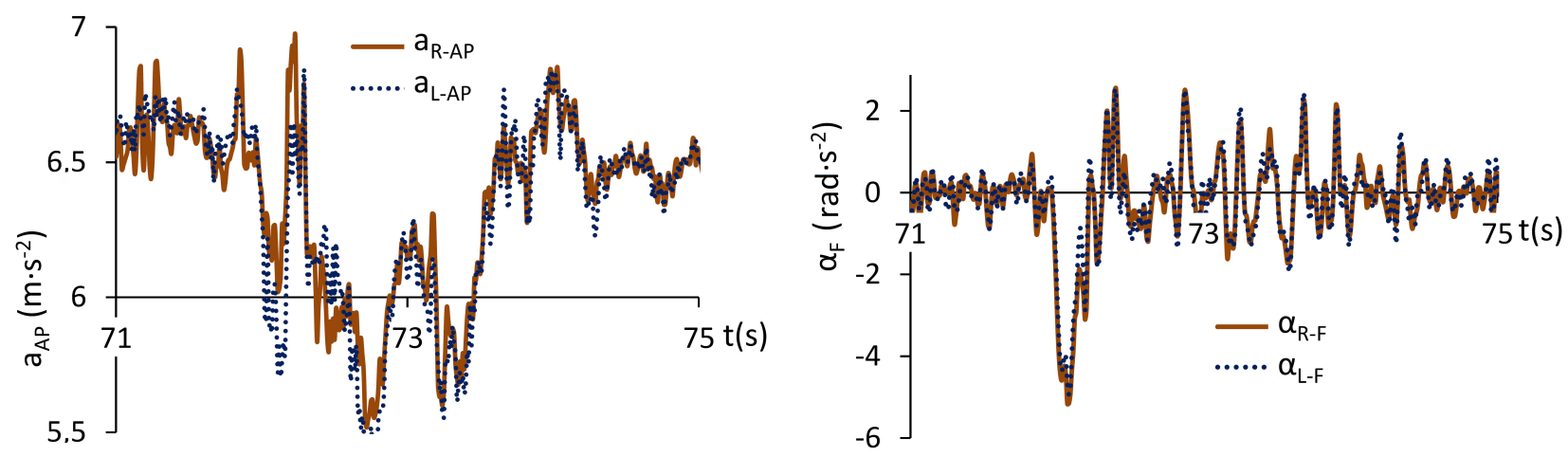

FigURE 6. Linear accelerations chart (a) in the anterior-posterior (AP) direction and angular accelerations $(\alpha)$ in the frontal plane $(\mathrm{F})$ of the left $(\mathrm{L})$ and right $(\mathrm{R})$ labyrinth of the vestibular system measured within the deceleration phase of unilateral rotation on the rotation chair.

\section{Results}

After realization of repeated HIT and unilateral rotation on the rotational chair, time dependencies of two linear accelerations in the anterior-posterior (aAP) direction for the left $(\mathrm{aL}-\mathrm{AP})$ and right (aRAP) labyrinth of the vestibular system were used, as well as two angular accelerations of roll movement in the frontal plane $(\alpha \mathrm{F})$ for the left $(\alpha \mathrm{L}-\mathrm{F})$ and right $(\alpha \mathrm{R}-\mathrm{F})$ labyrinth of the vestibular system, see Fig. 5 and Fig. 6. With regard to the clarity and easier comparison of the record, the linear accelerations and angular accelerations were depicted in one chart and simultaneously shorter sequels of the entire record were presented in Fig. 5 and Fig. 6. Fig. 5 shows four subsequent HIT tests. Fig. 6 shows the deceleration phase of unilateral rotation on the rotating chair where it is possible to assume an occurrence of acceleration and deceleration, respectively.

So called bilateral diagrams of mutual dependence of development of identical kinematic quantities were furthermore employed, only for the right and left part of a body. After the repeated HIT and unilateral rotation on the rotating chair a parallel record of two linear accelerations in the anterior-posterior direction, as well as two angular accelerations of roll movements in the frontal plane for the right and left labyrinth of the vestibular system, see Fig. 7 and Fig. 8 .

In the case of HIT the value of the correlation coefficient between the linear acceleration in the anteriorposterior direction of the right labyrinth of the vestibular system and the linear acceleration in the anteriorposterior acceleration of the left labyrinth of the vestibular system was 0.23 . In the case of HIT, the value of the correlation coefficient between the angular acceleration of the roll movement of the right labyrinth of the vestibular system and the angular acceleration of the roll movement of the left labyrinth of the vestibular system was 0.98 .

In the case of the unilateral rotation on the rotation chair, the value of the correlation coefficient between linear acceleration in the anterior-posterior direction of the right labyrinth of the vestibular system and linear acceleration in the anterior-posterior direction of the right labyrinth of the vestibular system was 0.86 . In the case of HIT, the value of the correlation coefficient between the angular acceleration of the roll movement of the right labyrinth of the vestibular system and the angular acceleration of the roll movement of the left labyrinth of the vestibular system was 0.78 . 

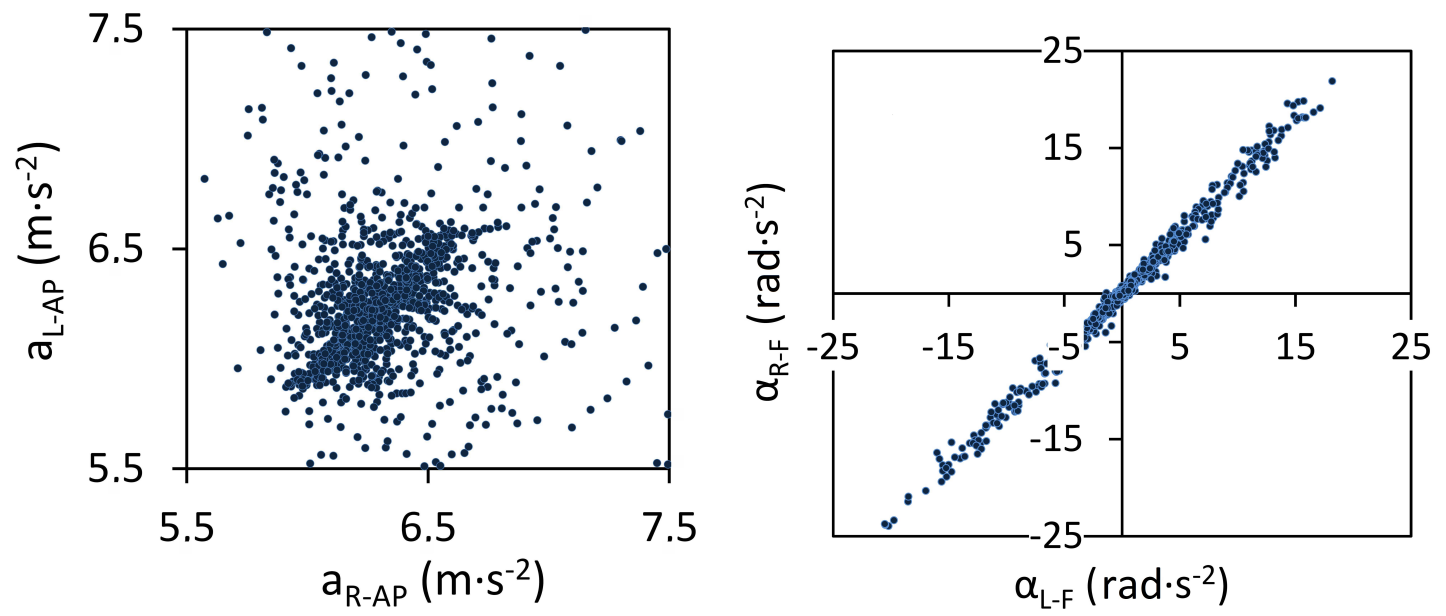

Figure 7. Chart of mutual dependencies of linear acceleration (a) in the anterior-posterior (AP) direction and mutual dependencies of angular accelerations $(\alpha)$ in the frontal plane $(\mathrm{F})$ of the left $(\mathrm{L})$ and the right $(\mathrm{R})$ labyrinth of the vestibular system measured within HIT.
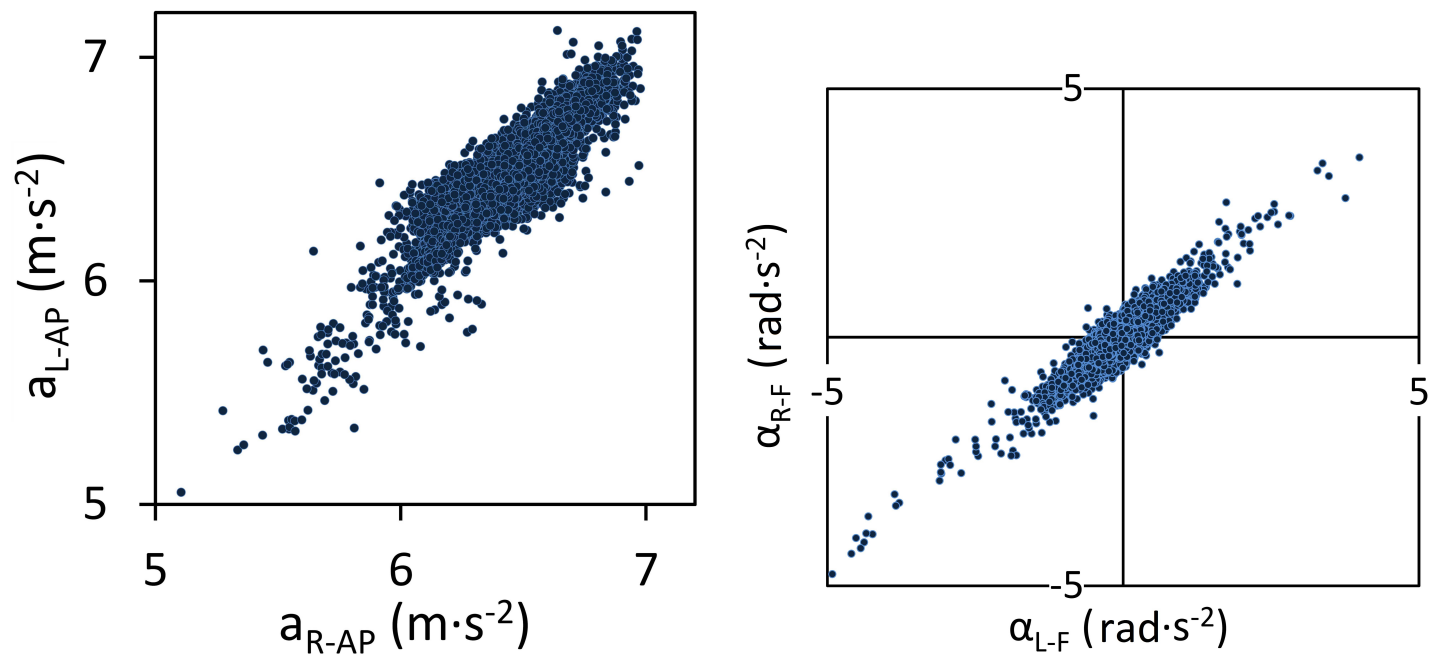

FiguRE 8. Chart of mutual dependencies of linear accelerations (a) in the anterior-posterior (AP) direction and mutual dependencies of angular accelerations $(\alpha)$ in the frontal plane $(\mathrm{F})$ of the left $(\mathrm{L})$ and right $(\mathrm{R})$ labyrinth of the vestibular system measured within the unilateral rotation on the rotating chair.

\section{Discussion}

A special system for measuring kinematic quantities of translational and rotational movement of the left and right labyrinth of the vestibular system has been proposed. It was found that there is no similar device enabling to measure the movement of the vestibular system specifically within the scope of its coordinate system. The system suitability test was carried out during unilateral assessment on the rotation chair and HIT. The results of the system testing show that the measured kinematic data for the right and left labyrinth of the vestibular system vary slightly, see Fig. 5 Fig. 6

In the case of accelerations of translational movements during HIT, the recorded development of accelerations of the left and right labyrinth of the vestibular system was even reverse. With the angular accelerations in HIT, such trend has not been recorded. The bilateral diagrams show, in the case of the translation accelerations, a significant dispersion of the points, from the hypothetically perfect symmetrical development of the values (lying on the hypothetical axis with an angle of $45^{\circ}$ ). With the angular acceleration in HIT, the dispersion of the values is minor and the recorded data are almost aligned. This stems from the fact, that within the HIT test, there is a complex 3-D movement in which the originally assumed rotation around the axis of the symmetry of a head, or longitudinal axis does not appear. However there is an occurrence of rotation around a point outside an axis which varies according to the action intensity of the examining person on the subject during HIT. There is a parallel occurrence of the rotation on all three planes. For this reason the entire translational acceleration measured using accelerometers is the sum of both acceleration of the translational movement, and peripheral acceleration during HIT. Both linear and angular accelerations vary during the above mentioned 
complex movement of the left and right labyrinth of the vestibular system. The stated differences in the measured accelerations are also confirmed by the low correlation of the record of the left and right labyrinth of the vestibular system which is only weak. In the case of the measured angular roll accelerations of the rotation movement, Fig. 7] the accelerations coincide highly, hence the presumption that with the HIT with the intention of movement around the vertical axis, a head, in the places of the left and right labyrinth of the vestibular system in the frontal place, rolls with similar angular acceleration. This also shows strong correlations between the data for the left and right labyrinth of the vestibular system which is strong.

Also in the case of the results of the measurement of accelerations during unilateral rotation on the rotation chair, the differences in the records of the left and right labyrinth of the vestibular system are apparent. Nevertheless, the symmetry corresponding with strong correlation is obvious by translational and rotational accelerations. In such a case, the similarity of the values stems from the direction of rotation, when the rotation around the vertical axis is always preserved by such positioning of the proband on the rotation chair that eliminates combined rotation in all planes as it occurred in the case of HIT. Nonetheless, even here we can see a slight difference since this is again not the rotation around the axis of the symmetry of a head but the rotation axis lies outside the symmetry axis. From the results it is evident that our proposed system allows recording kinematic quantities of the movement of the left and right labyrinth of the vestibular system which can differ. The practical usability has been thus proved above all for the medical practice and research where it is necessary to detect movement data of the left and right labyrinth of the vestibular system separately.

\section{Conclusions}

The proposed system differs from the traditional systems in the aspect of not using solely one gyroaccelerometric sensor which is not placed in the accurate anatomical position of the vestibular system. It employs a set of sensors allowing for the monitoring of the movements of the parts of the vestibular system within the scope of their coordinate systems. The presented device is designed mainly for application in clinical neurology with the aim to allow physicians to measure all linear and angular accelerations of labyrinths of the vestibular systems in medical examinations such as unilateral examination on the rotation chair, pulse rotation test and vestibular autorotation test. The system allows for a direct correlation of information on movements of the labyrinths of the vestibular systems in neurological assessments. Further utilization is possible in all cases where it is necessary to determine linear and angular acceleration of parts of the vestibular system, mainly in biomechanics. The subsequent research will be aimed at the proposal of methods of complex evaluation of 3-D movement for clinical practice with the intention to use the proposed methods for clinical examinations.

\section{ACKNOWLEDGEMENTS}

This work was supported by the Grant Agency of the Czech Technical University in Prague, grants SGS14/099/OHK4/1T/17 and SGS15/112/OHK4/1T/17.

\section{REFERENCES}

[1] R. Cerny, K. Strohm, J. Hozman, et al. Head in spacenoninvasive measurement of head posture. In Proceedings of the 11th Danube Symposium-International Otorhinolaryngological Congress, pp. 39-42. 2006.

[2] F. Horak, C. Jones-Rycewicz, F. O. Black, A. ShumwayCook. Effects of vestibular rehabilitation on dizziness and imbalance. Otolaryngology-head and neck surgery: official journal of American Academy of OtolaryngologyHead and Neck Surgery 106(2):175-180, 1992.

[3] J. Hejda, P. Kutilek, J. Hozman, R. Cerny. Motion capture camera system for measurement of head and shoulders position. Biomedical

Engineering/Biomedizinische Technik 57(SI-1

Track-B):472-475, 2012. DOI:10.1515/bmt-2012-4123

[4] J. Hozman, D. Sturm, J. Stoklasa. Measurement of head position in neurological practice. Biomedical Engineering [CD-ROM] Acta Press pp. 586-589, 2004.

[5] O. Kremmyda, H. Kirchner, S. Glasauer, et al. Falsepositive head-impulse test in cerebellar ataxia. Frontiers in neurology 3, 2012. DOI:10.3389/fneur.2012.00162.

[6] E. A. Keshner. Head-trunk coordination during linear anterior-posterior translations. Journal of Neurophysiology 89(4):1891-1901, 2003. DOI:10.1152/jn.00836.2001

[7] Y. Danilov, M. Tyler, K. Skinner, et al. Efficacy of electrotactile vestibular substitution in patients with peripheral and central vestibular loss. Journal of vestibular research: equilibrium $\&$ orientation 17(2-3):119, 2007. DOI:10.1109/iembs.2006.260899

[8] M. Higgins, P. D. Halstead, L. Snyder-Mackler, D. Barlow. Measurement of impact acceleration: mouthpiece accelerometer versus helmet accelerometer. Journal of athletic training 42(1):5, 2007. DOI:10.1109/iembs.2006.260899

[9] K. Nie, L. Ling, S. M. Bierer, et al. An experimental vestibular neural prosthesis: design and preliminary results with rhesus monkeys stimulated with modulated pulses. Biomedical Engineering, IEEE Transactions on 60(6):1685-1692, 2013. DOI:10.1109/tbme.2013.2241433

[10] J. O. Phillips, S. J. Shepherd, A. L. Nowack, et al. Longitudinal performance of a vestibular prosthesis as assessed by electrically evoked compound action potential recording. In Engineering in Medicine and Biology Society (EMBC), 2012 Annual International Conference of the IEEE, pp. 6128-6131. IEEE, 2012. DOI:10.1109/embc.2012.6347392.

[11] A. Clarke, A. Engelhorn, C. Hamann, U. Schönfeld. Measuring the otolith-ocular response by means of unilateral radial acceleration. Annals of the New York Academy of Sciences 871(1):387-391, 1999. DOI:10.1111/j.1749-6632.1999.tb09201.x 
[12] P. Cappa, F. Patanè, S. Rossi. A redundant accelerometric cluster for the measurement of translational and angular acceleration and angular velocity of the head. Journal of Medical Devices 1(1):14-22, 2007. DOI:10.1115/1.2355685

[13] V. Socha, P. Kutilek, O. Cakrt, R. Cerny. Comparative measurements of head angular movements using a camera system and a gyroscope system. Acta Polytechnica 54(4):295-300, 2014. DOI:10.14311/ap.2014.54.0295

[14] E. Keshner, Y. Dhaher. Characterizing head motion in three planes during combined visual and base of support disturbances in healthy and visually sensitive subjects. Gait \& posture 28(1):127-134, 2008. DOI:10.1016/j.gaitpost.2007.11.003

[15] T. Okada, E. Grunfeld, J. Shallo-Hoffmann, A. Bronstein. Vestibular perception of angular velocity in normal subjects and in patients with congenital nystagmus. Brain 122(7):1293-1303, 1999. DOI:10.1093/brain/122.7.1293

[16] H. G. MacDougall, I. S. Curthoys. Plasticity during vestibular compensation: the role of saccades. Frontiers in neurology 3, 2012. DOI:10.3389/fneur.2012.00021.

[17] C. C. Della Santina, P. D. Cremer, J. P. Carey, L. B. Minor. Comparison of head thrust test with head autorotation test reveals that the vestibulo-ocular reflex is enhanced during voluntary head movements. Archives of Otolaryngology-Head \& Neck Surgery 128(9):10441054, 2002. DOI:10.1001/archotol.128.9.1044.

[18] J. P. Carey, C. C. Della Santina. Principles of applied vestibular physiology. Cummings: Otolaryngology: Head $\&$ Neck Surgery 4th ed Philadelphia, PA: Elsevier Mosby 3:3115Y59, 2005. DOI:10.1016/b978-0-323-05283-2.00164-6

[19] R. E. Kalman. A new approach to linear filtering and prediction problems. Journal of Fluids Engineering 82(1):35-45, 1960. DOI:10.1115/1.3662552

[20] H. J. Luinge. Inertial sensing of human movement. Twente University Press, 2002.

[21] C. P. López. Symbolic differential and integral calculus. In MATLAB Differential Equations, pp. 125171. Springer, 2014. DOI:10.1007/978-1-4842-0310-1_8.

[22] P. Kutilek, S. Viteckova, Z. Svoboda, P. Smrcka. Kinematic quantification of gait asymmetry in patients with peroneal nerve palsy based on bilateral cyclograms. Journal of musculoskeletal $\&$ neuronal interactions 13(2):244-250, 2013. DOI:10.1007/978-3-319-00846-2_29

[23] W. W. Daniel, et al. Applied nonparametric statistics. PWS-Kent Boston, 1990. 